\title{
Time-Efficient and Cost-Effective Network Hardening Using Attack Graphs
}

\author{
Massimiliano Albanese*, Sushil Jajodia*†, and Steven Noel* \\ ${ }^{*}$ Center for Secure Information Systems \\ George Mason University \\ 4400 University Drive, Fairfax, VA 22030 \\ Email: \{malbanes,jajodia,snoel\}@gmu.edu \\ ${ }^{\dagger}$ The MITRE Corporation \\ 7515 Colshire Drive, McLean, VA 22102-7539
}

\begin{abstract}
Attack graph analysis has been established as a powerful tool for analyzing network vulnerability. However, previous approaches to network hardening look for exact solutions and thus do not scale. Further, hardening elements have been treated independently, which is inappropriate for real environments. For example, the cost for patching many systems may be nearly the same as for patching a single one. Or patching a vulnerability may have the same effect as blocking traffic with a firewall, while blocking a port may deny legitimate service. By failing to account for such hardening interdependencies, the resulting recommendations can be unrealistic and far from optimal. Instead, we formalize the notion of hardening strategy in terms of allowable actions, and define a cost model that takes into account the impact of interdependent hardening actions. We also introduce a nearoptimal approximation algorithm that scales linearly with the size of the graphs, which we validate experimentally.
\end{abstract}

Keywords-network hardening, vulnerability analysis, attack graphs, intrusion prevention, reliability.

\section{INTRODUCTION}

Attackers can leverage the complex interdependencies of network configurations and vulnerabilities to penetrate seemingly well-guarded networks. In-depth analysis of network vulnerabilities must consider attacker exploits not merely in isolation, but in combination. Attack graphs reveal such threats by enumerating potential paths that attackers can take to penetrate networks. This helps determine whether a given set of network hardening measures provides safety of given critical resources.

Attack graph analysis can be extended to automatically generate recommendations for hardening networks. One must consider combinations of network conditions to harden, which has corresponding impact on removing paths in the attack graph. Further, one can generate hardening solutions that are optimal with respect to some notion of cost. Such hardening solutions prevent the attack from succeeding, while minimizing the associated costs.

However, as we show, the general solution to optimal network hardening scales exponentially as the number of

The work presented in this paper is supported in part by the Army Research Office under MURI award number W911NF-09-1-0525, and by the Office of Naval Research under award number N00014-12-1-0461. hardening options itself scales exponentially with the size of the attack graph. In applying network hardening to realistic network environments, it is crucial that the algorithms are able to scale. Progress has been made in reducing the complexity of attack graph manipulation so that it scales quadratically (linearly within defined security zones) [1]. However, previous approaches for generating hardening recommendations search for exact solutions [2], which is an intractable problem.

Another limitation of previous work is the assumption that network conditions are hardened independently. This assumption does not hold true in real network environments. Realistically, network administrators can take actions that affect vulnerabilities across the network, such as pushing patches out to many systems at once. Further, the same hardening result may be obtained through more than one action. Overall, to provide realistic recommendations, our hardening strategy must take such factors into account.

We remove the assumption of independent hardening actions. Instead, we define a network hardening strategy as a set of allowable atomic actions that involve hardening multiple network conditions. We introduce a formal cost model that accounts for the impact of these hardening actions. This allows the definition of hardening costs that accurately reflect realistic network environments. Because computing the minimum-cost hardening solution is intractable, we introduce an approximation algorithm for optimal hardening. This algorithm finds near-optimal solutions while scaling almost linearly - for certain values of the parameters - with the size of the attack graph, which we validate experimentally. Finally, we determine the theoretical upper bound for the worst-case approximation ratio, and show that, in practice, the approximation ratio is much lower than such bound.

The paper is organized as follows. Section II discusses related work. Section III recalls some preliminary definitions, whereas Section IV provides a motivating example. Then Section V introduces the proposed cost model, and Section VI describes our approach to time-efficient and costeffective network hardening. Finally, Section VII reports experimental results, and Section VIII gives some concluding remarks and indicates further research directions. 


\section{RELATED WORKS}

A number of tools are available for scanning network vulnerabilities, such as Nessus [3], but these only report isolated vulnerabilities. Attack graphs are constructed by analyzing the inter-dependencies between vulnerabilities and security conditions that have been identified in the target network [4], [5], [6], [7], [8], [9], [10], [11], [12], [13]. Such analysis can be either forward, starting from the initial state [8], [12] or backward from the goal state [9], [11]. Model checking was first used to analyze whether the given goal state is reachable from the initial state [9], [14], but later used to enumerate all possible sequences of attacks between the two states [11], [15].

The explicit attack sequences produced by a model checker face a serious scalability issue, because the number of such sequences is exponential in the number of vulnerabilities multiplied by the number of hosts. To avoid such combinatorial explosion, a more compact representation of attack graphs was proposed in [4]. The monotonicity assumption underlies this representation, i.e., an attacker never relinquishes any obtained capability. This newer representation can thus keep exactly one vertex for each exploit or security condition, leading to an attack graph of polynomial size (in the total number of vulnerabilities and security conditions).

Attack graphs have also been used for correlating intrusion alerts into attack scenarios [16], [17]. Such alert correlation methods are parallel to our work, because they aim to employ the knowledge encoded in attack graphs for detecting and taking actions against actual intrusions, whereas our work aims to harden the network before any intrusion may happen.

Algorithms exist to find the set of exploits from which the goal conditions are reachable [4]. This eliminates some irrelevant exploits from further consideration because they do not contribute to reaching the goal condition. The minimal critical attack set is a minimal set of exploits in an attack graph whose removal prevents attackers from reaching any of the goal states [4], [11], [15]. The minimal critical attack set thus provides solutions to harden the network. However, these methods ignore the critical fact that consequences cannot be removed without removing the causes. The exploits in the solution usually depend on other exploits that also need to be disabled. The solution is thus not directly enforceable. Moreover, after taking into account those implied exploits the solution is no longer minimum.

A more effective solution to automate the task of hardening a network against multi-step intrusions was proposed by Wang et al. in [2]. Unlike previous approaches, which require removing exploits, this solution focuses on initially satisfied conditions only. Initial conditions can be disabled, leading to a readily deployable solution. However, Wang et al. assumed that initial conditions can be independently disabled. Although this is true in some cases, there may exist dependencies between initial conditions, such that removing certain initial conditions may also disable additional conditions, and this might not be necessary to harden the network. The work presented in this paper differs significantly from previous work in that we (i) drop the assumption that initial conditions can be independently disabled; (ii) introduce a formal cost model; and (iii) present an approximation algorithm that generates suboptimal solutions efficiently.

\section{PRELIMINARIES}

Attack graphs represent prior knowledge about vulnerabilities, their dependencies, and network connectivity. Two different representations are possible for an attack graph. First, an attack graph can explicitly enumerate all possible sequences of vulnerabilities an attacker can exploit to reach a target, i.e., all possible attack paths. However, such graphs face a combinatorial explosion in the number of attack paths. Second, with a monotonicity assumption stating an attacker never relinquishes obtained capabilities, an attack graph can record the dependencies among vulnerabilities and keep attack paths implicitly without losing any information. The resulting attack graph has no duplicate vertices and hence has a polynomial size in the number of vulnerabilities multiplied by the number of connected pairs of hosts.

In this paper, we adopt the definition of attack graph presented in [17], which assumes the latter notion of attack graphs.

Definition 1 (Attack graph): Given a set of exploits $E$, a set of security conditions $C$, a require relation $R_{r} \subseteq C \times E$, and an imply relation $R_{i} \subseteq E \times C$, an attack graph $G$ is the directed graph $G=\left(E \cup C, R_{r} \cup R_{i}\right)$, where $E \cup C$ is the vertex set and $R_{r} \cup R_{i}$ the edge set.

We denote an exploit as a predicate $v\left(h_{s}, h_{d}\right)$, indicating an exploitation of vulnerability $v$ on the destination host $h_{d}$, initiated from the source host $h_{s}$. Similarly, we write $v(h)$ for exploits involving only local host $h$.

A security condition is a predicate $c\left(h_{s}, h_{d}\right)$ that indicates a satisfied security-related condition $c$ involving the source host $h_{s}$ and the destination host $h_{d}$ (when a condition involves a single host, we simply write $c(h)$ ). Examples of security conditions include the existence of a vulnerability on a given host or the connectivity between two hosts. Initial conditions are a special subset of security conditions, as defined below [2].

Definition 2 (Initial conditions): Given an attack graph $G=\left(E \cup C, R_{r} \cup R_{i}\right)$, initial conditions refer to the subset of conditions $C_{i}=\left\{c \in C \mid \nexists e \in E\right.$ s.t. $\left.(e, c) \in R_{i}\right\}$, whereas intermediate conditions refer to the subset $C \backslash C_{i}$.

Intermediate conditions are usually consequences of exploits and hence cannot be disabled without removing the causes. Instead, initial conditions are not created through the execution of exploits, thus they might be removed. Without loss of generality, we will explicitly model only initial conditions that can be disabled, and omit initial 


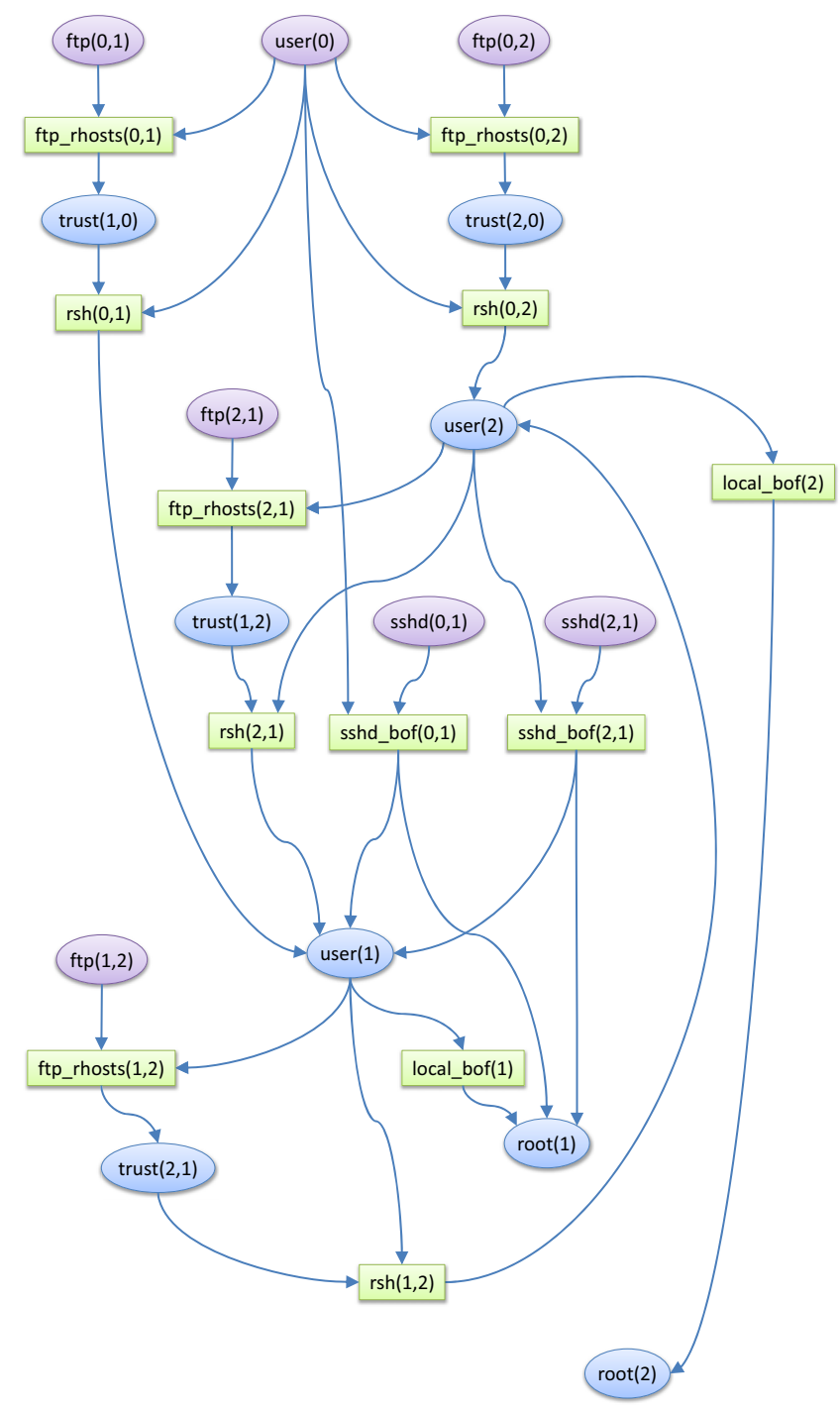

Figure 1. An example of attack graph, including initial conditions (purple ovals), exploits (green rectangles), and intermediate conditions (blue ovals)

conditions that network administrators cannot control, such as privileges on the attacker's machine.

\section{Motivating Example}

Figure 1 depicts an example of attack graph, with exploits appearing as rectangles and conditions as ovals. Purple ovals represent initial conditions, whereas blue ovals represent intermediate conditions. Some modeling simplifications have been made, such as combining transport-layer ftp connectivity between two hosts $h_{s}$ and $h_{d}$, physical-layer connectivity, and the existence of the ftp daemon on host $h_{d}$ into a single condition $f t p\left(h_{s}, h_{d}\right)$. In this example, we assume that our objective is to harden the network with respect to target condition root(2), i.e., we want to prevent the attacker from gaining root privileges on host 2 . The scenario depicted in
Figure 1 is relatively simple, with three hosts - denoted host 0,1 , and 2 respectively - and four types of vulnerabilities - ftp_rhosts, rsh, sshd_bof, and local_bof. However, because multiple interleaved attack paths can lead to the goal condition, an optimal solution to harden the network is still not apparent from the attack graph itself, and finding such a solution by hand may not be trivial. As an example of attack path, the attacker can first establish a trust relationship from his machine (host 0 ) to host 2 (condition trust $(2,0)$ ) via the exploit ftp_rhosts $(0,2)$ on host 2 , then gain user privileges on host 2 (condition user $(2)$ ) with an rsh login (exploit $r \operatorname{sh}(0,2)$ ), and finally achieve the goal condition $\operatorname{root}(2)$ using a local buffer overflow attack on host 2 (exploit local_bof(2)). The following are some of the valid attack paths that can be generated using existing algorithms [4].

- ftp_rhosts $(0,2), \operatorname{rsh}(0,2)$, local_bof $(2)$

- ftp_rhosts $(0,1)$, rsh $(0,1)$, ftp_rhosts $(1,2), \operatorname{rsh}(1,2)$, local_bof $(2)$

- $s s h d \_b o f(0,1), f t p \_r h o s t s(1,2), r s h(1,2)$, local_bof $(2)$

Intuitively, to prevent the goal condition from being satisfied, a solution to network hardening must break all the attack paths leading to the goal. This intuition was captured by the concept of critical set, that is, a set of exploits (and corresponding conditions) whose removal from the attack graph will invalidate all attack paths. It has also been shown that finding critical sets with the minimum cardinality is NP-hard, whereas finding a minimal critical set (that is, a critical set with no proper subset being a critical set) is polynomial. Based on the above attack paths, there are many minimal critical sets, such as $\{r \operatorname{sh}(0,2), r s h(1,2)\}, \quad\left\{f t p \_r h o s t s(0,2), r s h(1,2)\right\}$, $\left\{f t p \_r h o s t s(1,2), r \operatorname{sh}(0,2)\right\}$, and so on. If any of those sets of exploits could be completely removed, all the attack paths would become invalid, and hence the target condition would be unreachable. Unfortunately, the above solution ignores the following important fact. Not all exploits are under the direct control of administrators. An exploit can only be removed by disabling its required conditions, but not all conditions can be disabled at will. Intuitively, a consequence cannot be removed without removing its causes. Some conditions are implied by other exploits. Such intermediate conditions cannot be independently disabled without removing the exploits that imply them. Only those initial conditions that are not implied by any exploit can be disabled independently of other exploits. Hence, it is important to distinguish between these two kinds of conditions, as formalized in Definition 2.

For instance, in Figure 1, exploit $r \operatorname{sh}(1,2)$ cannot be independently removed, because the two conditions it requires, trust $(2,1)$ and user $(1)$, are both intermediate conditions and cannot be independently disabled. As long as an attacker can satisfy those two conditions through other exploits (for example, ftp_rhosts $(1,2)$ and sshd_bof $(2,1)$ ), 
the realization of the exploit $r \operatorname{sh}(1,2)$ is unavoidable. Hence, any of the above minimal critical sets, such as $\{r \operatorname{sh}(0,2), r \operatorname{sh}(1,2)\}$, is theoretically a sound solution, but practically not enforceable. For this reason, the approach proposed in [2] relies on initial conditions only. However, it has some limitations that we address in this paper.

The approach of [2] has no explicit cost model and assumes that each initial condition can be independently disabled. Thus, even when all possible solutions are enumerated, determining the one with the minimum cost is based either on a qualitative notion of cost or on simply counting the conditions that need to be disabled. For the attack graph of Figure 1, the algorithm in [2] returns two solutions, $\{f t p(0,2), f t p(1,2)\}$ and $\{f t p(0,2), f t p(0,1), \operatorname{sshd}(0,1)\}^{1}$. At this point, there is no clear procedure to decide which solution has the minimum cost, unless we make the assumption that the cost of removing each individual condition is assigned by administrators.

Intuitively, one may expect the solution $\{f t p(0,2)$, $f t p(1,2)\}$ to have a lower cost than $\{f t p(0,2), f t p(0,1)$, $\operatorname{ssh}(0,1)\}$, as fewer conditions need to be disabled. However, removing both $f t p(0,2)$ and $f t p(1,2)$ may only be possible if the ftp service on host 2 is shut down. This action may have a considerable cost in terms of disruption to legitimate users. In this case, the combined cost of removing the conditions $\{f t p(0,2), f t p(0,1), \operatorname{sshd}(0,1)\}$ may be lower, as it may be achieved by simply blocking all traffic from host 0 .

To conclude, note that the attack graph of Figure 1 has the same hardening solutions as the simplified attack graph of Figure 2. This is possible because the algorithm in [2] traverses the graph from target conditions to initial conditions, and, relying on the monotonicity assumption, breaks all the cycles. Intuitively, from the point of view of a target condition, the attack graph can be seen as a tree rooted at the target condition and having initial conditions as the leaf nodes. In fact, each condition is implied by one or more exploits. In turn, each exploit requires one or more preconditions to be satisfied. We leverage this observation in our approach to network hardening.

\section{Cost Model}

Disabling a set of initial conditions in order to prevent attacks on given targets may result in undesired effects, such as denial of service to legitimate users. These effects are greatly amplified when initial conditions cannot be individually disabled, but rather require actions that disable a larger number of conditions. In the following, we define a network hardening strategy as a set of atomic actions that can be taken to harden a network.

\footnotetext{
${ }^{1}$ Initial conditions that the administrators cannot control are not considered for the purpose of network hardening. In the example of Figure 1, the condition user(0), corresponding to user privileges on the attacker's machine, is ignored.
}

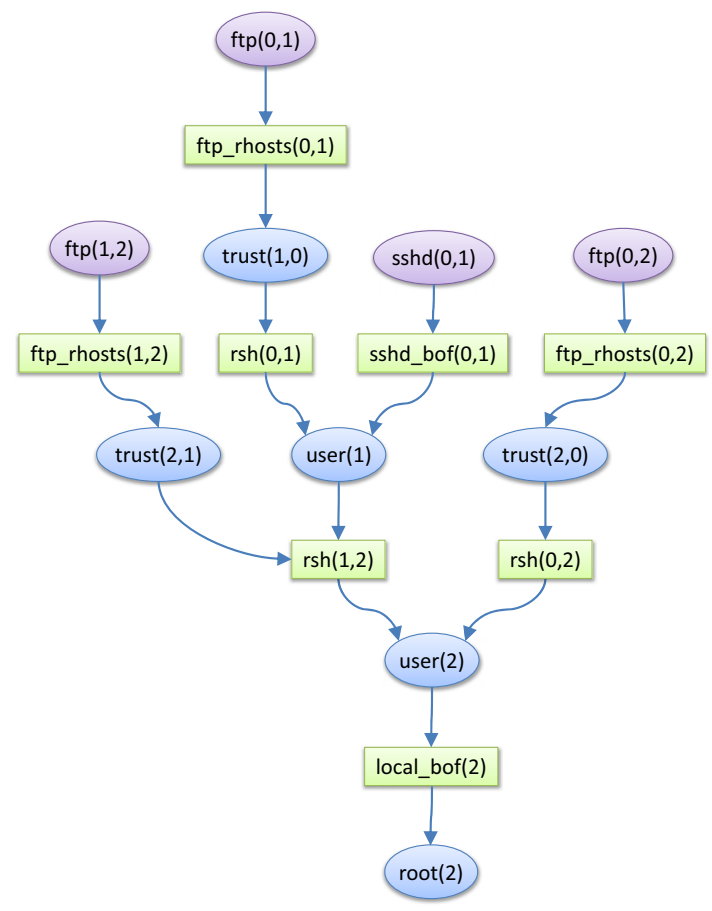

Figure 2. A tree-style attack graph equivalent to the graph of Figure 1 w.r.t. target condition $\operatorname{root}(2)$

For instance, an allowable hardening action may consist in stopping ftp service on a given host. Thus, each action may have additional effects besides disabling a desired condition. Such effects must be taken into account when computing minimum-cost solutions. Previous work simply assumes that initial conditions can be individually disabled. We take a more general approach and therefore drop this assumption. For instance, in the attack graph of Figure 1, disabling $f t p(1,2)$ might not be possible without also disabling $\operatorname{ftp}(0,2)$.

Definition 3 (Allowable hardening action): Given an attack graph $G=\left(E \cup C, R_{r} \cup R_{i}\right)$, an allowable hardening action (or simply hardening action) $A$ is any subset of the set $C_{i}$ of initial conditions such that all the conditions in $A$ can be jointly disabled in a single step, and no other initial condition $c \in C_{i} \backslash A$ is disabled when conditions in $A$ are disabled.

A hardening action $A$ is said to be minimal if and only if $\nexists A^{*} \subset A$ s.t. $A^{*}$ is an allowable hardening action. We use $\mathcal{A}$ to denote the set of all possible hardening actions.

Figure 3 depicts the same attack graph of Figure 2, but it explicitly shows the allowable hardening actions, represented as rounded rectangles. Dashed edges indicate which conditions are disabled by each action. Intuitively, a network hardening action is an atomic step that network administrators can take to harden the network (e.g., closing an ftp port). When an action $A$ is taken, all and only 


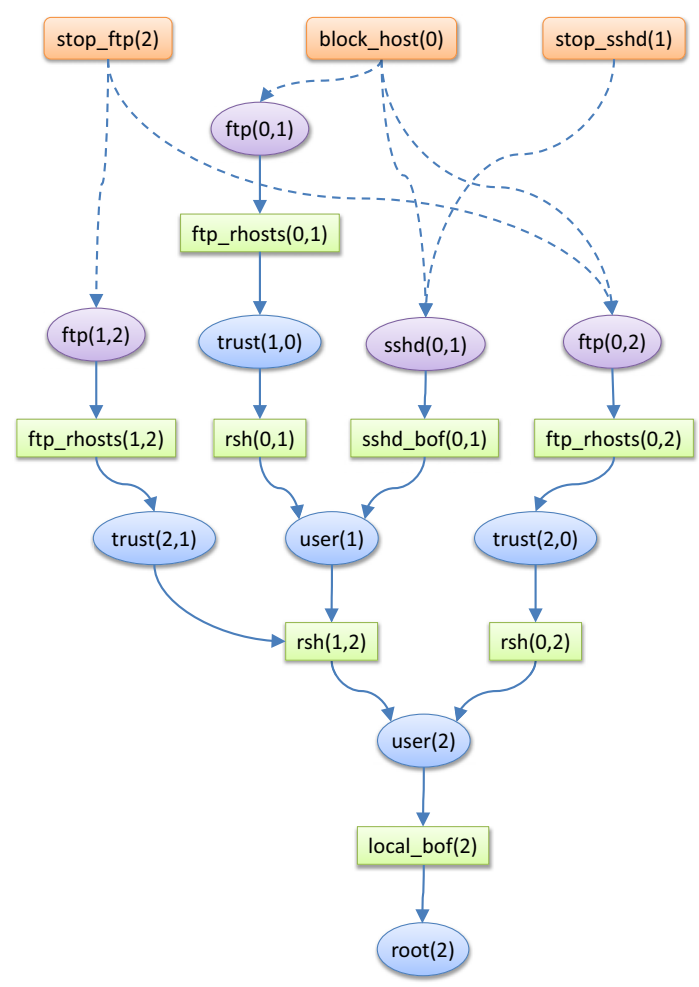

Figure 3. Possible hardening actions (orange rectangles) for the attack graph of Figure 2

the conditions in $A$ are removed ${ }^{2}$. In the example of Figure $3, \mathcal{A}=\{$ stop_ftp $(2)$, block_host(0), stop_sshd(1) $\}$, stop_ftp $(2)=\{f t p(0,2), f t p(1,2)\}$, block_host $(0)=$ $\{f t p(0,1), \operatorname{sshd}(0,1), f t p(0,2)\}$, and stop_sshd $(1)=$ $\{\operatorname{sshd}(0,1)\}$. In this example, the condition $\operatorname{ftp}(1,2)$ cannot be individually disabled, and can only be disabled by taking action stop_ftp(2), which also disables $f t p(0,2)^{3}$.

Therefore, when choosing a set of initial conditions to be removed in order to prevent attacks on given targets, we should take into account all the implications of removing those conditions. Removing specific initial conditions may require to take actions that disable additional conditions, including conditions not explicitly modeled in the attack graph, such as conditions that are not part of any attack path. To address this problem, we formalize the notion of hardening strategy in terms of allowable actions, and define a cost model that takes into account the impact of hardening actions. This novel approach improves the state of the art, while preserving the key idea that solutions are truly enforceable only if they operate on initial conditions.

First, we drop the assumption that initial conditions can

\footnotetext{
${ }^{2}$ In practice, an action may also remove conditions not explicitly modeled in the attack graph, and this should be taken into account when assigning a cost to each action.

${ }^{3}$ More precisely, all conditions of the form $\operatorname{ftp}(x, 2)$, where $x$ is any host, are disabled by action stop_ftp(2).
}

be individually disabled. In our framework, this simplifying assumption corresponds to the special case where, for each initial condition, there exists an allowable action that disables that condition only, i.e., $\left(\forall c \in C_{i}\right)(\exists A \in \mathcal{A}) A=\{c\}$. We then define the notion of network hardening strategy in terms of allowable actions.

Definition 4 (Network hardening strategy): Given an attack graph $G=\left(E \cup C, R_{r} \cup R_{i}\right)$, a set $\mathcal{A}$ of allowable actions, and a set of target conditions $C_{t}=\left\{c_{1}, \ldots, c_{n}\right\}$, a network hardening strategy (or simply hardening strategy) $S$ is a set of network hardening actions $\left\{A_{1}, \ldots, A_{m}\right\}$ s.t. conditions $c_{1}, \ldots, c_{n}$ cannot be reached after all the actions in $S$ have been taken. We use $\mathcal{S}$ to denote the set of all possible strategies, and $C(S)$ to denote the set of all the conditions disabled under strategy $S$, i.e., $C(S)=\bigcup_{A \in S} A$.

Intuitively, a hardening strategy is a set of allowable actions breaking all attack paths leading to the target conditions.

We now introduce a cost model, enabling a more accurate analysis of available hardening options.

Definition 5 (Hardening cost function): A hardening cost function is any function cost $: \mathcal{S} \rightarrow \mathbb{R}^{+}$that satisfies the following axioms:

$$
\operatorname{cost}(\emptyset)=0
$$

$$
\begin{gathered}
\left(\forall S_{1}, S_{2} \in \mathcal{S}\right)\left(C\left(S_{1}\right) \subseteq C\left(S_{2}\right) \Rightarrow \operatorname{cost}\left(S_{1}\right) \leq \operatorname{cost}\left(S_{2}\right)\right) \\
\left(\forall S_{1}, S_{2} \in \mathcal{S}\right)\left(\operatorname{cost}\left(S_{1} \cup S_{2}\right) \leq \operatorname{cost}\left(S_{1}\right)+\operatorname{cost}\left(S_{2}\right)\right)
\end{gathered}
$$

In other words, the above definition requires that (i) the cost of the empty strategy - the one not removing any condition - is 0; (ii) if the set of conditions disabled under $S_{1}$ is a subset of the conditions disabled under $S_{2}$, then the cost of $S_{1}$ is less than or equal to the cost of $S_{2}$ (monotonicity); and (iii) the cost of the combined strategy $S_{1} \cup S_{2}$ is less than or equal to the sum of the individual costs of $S_{1}$ and $S_{2}$ (triangular inequality).

Combining the three axioms above, we can conclude that $\left(\forall S_{1}, S_{2} \in \mathcal{S}\right)\left(0 \leq \max \left(\operatorname{cost}\left(S_{1}\right), \operatorname{cost}\left(S_{2}\right)\right) \leq\left(\operatorname{cost}\left(S_{1} \cup\right.\right.\right.$ $\left.\left.S_{2}\right) \leq \operatorname{cost}\left(S_{1}\right)+\operatorname{cost}\left(S_{2}\right)\right)$.

A cost function is said to be additive if and only if the following additional axiom is satisfied.

$$
\begin{aligned}
\left(\forall S_{1}, S_{2} \in \mathcal{S}\right) \quad\left(S_{1} \cap S_{2}=\emptyset \Longleftrightarrow\right. & \\
& \left.\operatorname{cost}\left(S_{1}\right)+\operatorname{cost}\left(S_{2}\right)=\operatorname{cost}\left(S_{1} \cup S_{2}\right)\right)
\end{aligned}
$$

Many different cost functions may be defined. The following is a very simple cost function:

$$
\operatorname{cost}_{a}(S)=|C(S)|
$$

The above cost function simply counts the initial conditions that are removed under a network hardening strategy $S$, and clearly satisfies the three axioms of Definition 5. If actions in $\mathcal{A}$ are pairwise disjoint, then $\operatorname{cost}_{a}$ is also additive. 


\section{NETWORK HARDENING}

In this section, we first examine in more details the limitations of the approach proposed in [2], and then introduce our approximation algorithm to find reasonably good hardening strategies in a time efficient manner.

\section{A. Limitations of Previous Approach}

The algorithm presented in [2] starts from a set $C_{t}$ of target conditions and traverses the attack graph backwards, making logical inferences. At the end of the graph traversal, a logic proposition of the initial conditions is derived as the necessary and sufficient condition for hardening the network with respect to $C_{t}$. This proposition then needs to be converted to its disjunctive normal form (DNF), with each disjunction in the DNF representing a particular sufficient option to harden the network. Although the logic proposition can be derived efficiently, converting it to its DNF may incur into an exponential explosion.

Algorithm BackwardSearch (Algorithm 1) is functionally equivalent to the one described in [2] - in that it generates all possible hardening solutions ${ }^{4}$ - under the simplifying hypothesis that initial conditions can be individually disabled, i.e., $\left(\forall c_{i} \in C_{i}\right)(\exists A \in \mathcal{A})\left(A=\left\{c_{i}\right\}\right)$. However, our rewriting of the algorithm has several advantages over its original version. First, it is more general, as it does not assume that initial conditions can be individually disabled, and incorporates the notions of allowable action and hardening strategy defined in Section V. Second, it directly computes a set of possible hardening strategies, rather then a logic proposition that requires additional processing in order to provide actionable intelligence. Last, in a time-constrained or real-time scenario where one may be interested in the first available hardening solution, the rewritten algorithm can be easily modified to terminate as soon as a solution is found. To this aim, it is sufficient to change the condition of the main while loop (Line 3) to $(\nexists S \in \mathcal{S})\left(S \subseteq C_{i}\right)$. Such variant of the algorithm will generate hardening strategies that disable initial conditions closer to the target conditions. However, when used to find the minimum-cost hardening solution, Algorithm BackwardSearch still faces the combinatorial explosion described below. Instead, the algorithm introduced in Section VI-B provides a balance between the optimality of the solution and the time to compute it.

Under the simplifying hypothesis that initial conditions can be individually disabled - i.e., $\left(\forall c_{i} \in C_{i}\right)(\exists A \in$ $\mathcal{A})\left(A=\left\{c_{i}\right\}\right)-$ and allowable actions are pairwise disjoint - i.e., $\left(\forall A_{i}, A_{j} \in \mathcal{A}\right)\left(A_{i} \cap A_{j}=\emptyset\right)$ - it can be proved that, in the worst case, the number of possible hardening strategies is

$$
|\mathcal{S}|=\left|C_{t}\right| \cdot n^{\sum_{k=1}^{\frac{d}{2}} n^{k}}
$$

\footnotetext{
${ }^{4}$ For ease of presentation, the pseudocode of Algorithm 1 does not show how cycles are broken. This is done as in the original algorithm.
}

and the size of each solution is $n^{\frac{d}{2}}$, where $d$ is the maximum distance (number of edges) between initial and target conditions $^{5}$, and $n$ is the maximum in-degree of nodes in the attack graph. Worst case complexity is then $O\left(n^{n^{d}}\right)$. The proof is omitted for reasons of space.

The authors of [2] rely on the assumption that the attack graph of a small and well-protected network is usually small and sparse (the in-degree of each node is small), thus, even if the complexity is exponential, running time should be acceptable in practice. However, the result above shows that computing an optimal solution may be impractical even for relatively small attack graphs. For instance, consider the attack graph of Figure 4, where $n=2, C_{t}=\left\{c_{21}\right\}$, and $d=4$. According to Equation 6, there are 64 possible hardening strategies in the worst case, each of size 4 . The strategy that disables the set of initial conditions $\left\{c_{1}, c_{3}, c_{9}, c_{11}\right\}$ is one of such possible strategies. When $d=6$, the number of initial condition is 64 , and the number of possible strategies becomes 16,384 . For $d=8,\left|C_{i}\right|=256$ and the number of possible strategies is over a billion.

\section{B. Approximation Algorithm}

To address the limitations of the previous network hardening algorithm, we now propose an approximation algorithm that computes reasonably good solutions in a time efficient manner. We will show that, under certain conditions, the solutions computed by the proposed algorithm have a cost that is bound to be within a constant factor of the optimal cost.

Algorithm ForwardSearch (Algorithm 2) traverses the attack graph forward, starting from initial conditions. A key advantage of traversing the attack graph forward is that intermediate solutions are indeed network hardening strategies with respect to intermediate conditions. In fact, in a single pass, Algorithm ForwardSearch can compute hardening strategies with respect to any condition in $C$. To limit the exponential explosion of the search space, intermediate solutions can be pruned - based on some pruning strategy - whereas pruning is not possible for the algorithm that traverses the graph backwards. In fact, in this case, intermediate solutions may contain exploits and intermediate conditions, and we cannot say anything about their cost until all the exploits and intermediate conditions have been replaced with sets of initial conditions.

In this section, for ease of presentation, we consider hardening problems with a single target condition. The generalization to the case where multiple target conditions need to be hardened at the same time is straightforward and is discussed below.

Given a set $C_{t}$ of target conditions, we add a dummy exploit $e_{i}$ for each condition $c_{i} \in C_{t}$, such that $e_{i}$ has $c_{i}$ as its only precondition, as shown in Figure 5. Then, we add a

\footnotetext{
${ }^{5}$ Note that $d$ is always an even number.
} 

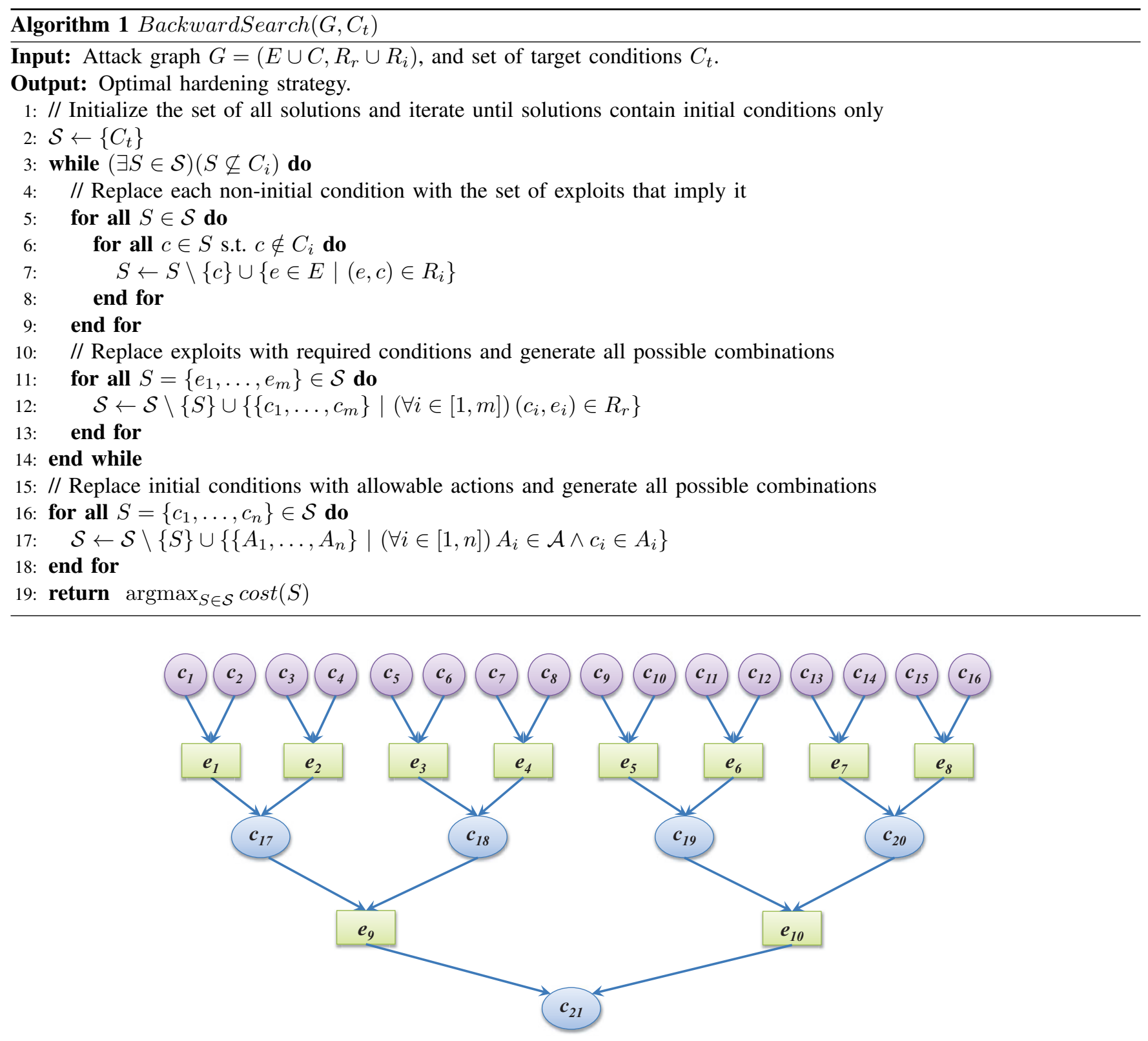

Figure 4. Example of attack graph with $n=2$ and $d=4$

dummy target condition $c_{t}$, such that all the dummy exploits $e_{i}$ have $c_{t}$ are their only postcondition. It is clear that any strategy that hardens the network with respect to $c_{t}$ implicitly hardens the network with respect to each $c_{i} \in C_{t}$. In fact, as $c_{t}$ is reachable from any dummy exploit $e_{i}$, all such exploits need to be prevented, and the only way to achieve this is by disabling the corresponding preconditions, that is hardening the network with respect to all target conditions in $C_{t}$.

Additionally, we assume that, given a target condition $c_{t}$, the attack graph is a tree rooted at $c_{t}$ and having initial conditions as leaf nodes. In Section IV, we showed an example of how this can be achieved using the mechanism to break cycle adopted by the algorithm in [2]. If the attack graph is not a tree, it can be converted to this form by using such mechanism. Looking at the attack graph from the point of view of a given target condition has the additional advantage of ignoring exploits and conditions that do not contribute to reaching that target condition.

On Line 1, the algorithm performs a topological sort of the nodes in the attack graph (exploits and security conditions), and pushes them into a queue, with initial conditions at the front of the queue. While the queue is not empty, an element 


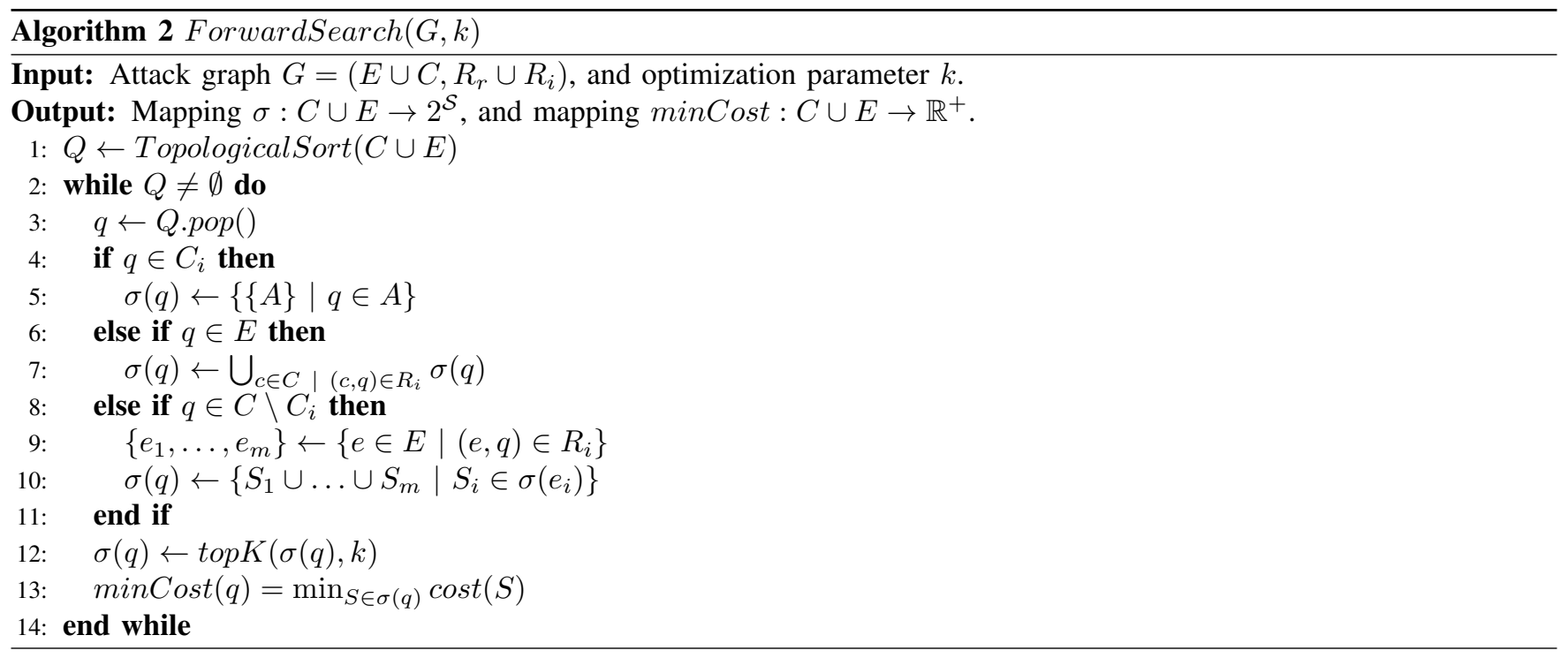

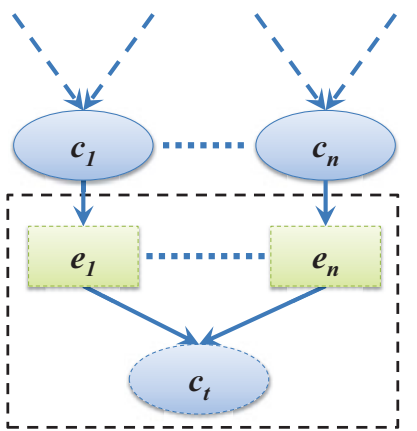

Figure 5. Example of multiple target conditions and dummy target

$q$ is popped from the queue. If $q$ is an initial condition, then $q$ is associated with a set of strategies $\sigma(q)$ such that each strategy simply contains one of the allowable actions in $\mathcal{A}$ disabling $q$ (Line 5). If $q$ is an exploit, then $q$ is associated with a set of strategies $\sigma(q)$ that is the union of the sets of strategies for each condition $c$ required by $q$ (Line 7). In fact, an exploit can be prevented by disabling any of its required conditions. Finally, if $q$ is an intermediate condition, then $q$ is associated with a set of strategies $\sigma(q)$ such that each strategy is the union of a strategy for each of the exploits that imply $q$ (Lines 9-10). In fact, in order to disable an intermediate condition, all the exploits that imply it must be prevented. When suboptimal solutions are acceptable, then only the best $k$ intermediate solutions are maintained at each step of the algorithm (Line 12), and the minimal hardening cost for the current node is computed accordingly (Line 13).

Example 1: Consider the attack graph of Figure 3. The only three allowable actions on the corresponding network are $s t o p \_f t p(2)=\{f t p(1,2), f t p(0,2)\}$, block_host $(0)=$ $\{f t p(0,1), \operatorname{sshd}(0,1), f t p(0,2)\}$, and stop_sshd $(1)=$ $\{\operatorname{sshd}(0,1)\}$. Assume that $\operatorname{cost}\left(\left\{\operatorname{stop\_ ftp}(2)\right\}\right)=20$, $\operatorname{cost}(\{$ block_host $(0)\})=10$, and $\operatorname{cost}\left(\left\{\operatorname{stop\_ sshd}(1)\right\}\right)=$ 15. It is clear that the optimal strategy to harden the network with respect to $\operatorname{root}(2)$ is $S=\left\{b l o c k \_h o s t(0)\right\}$, with a cost of 10. Let us now examine the behavior of the algorithm for $k=1$. All nodes are added to the queue in topological order, and initial conditions are examined first. After all the initial conditions have been examined, we obtain $\sigma(f t p(1,2))=\left\{\left\{\operatorname{stop} \_f t p(2)\right\}\right\}, \sigma(f t p(0,1))=$ $\{\{$ block_host $(0)\}\}, \sigma(\operatorname{sshd}(0,1))=\{\{$ block_host $(0)\}\}$, and $\sigma(f t p(0,2))=\{\{$ block_host $(0)\}\}$. Once the algorithm examines exploit $r \operatorname{sh}(1,2)$, on Line 7 , before pruning, we obtain $\sigma(r s h(1,2))=\left\{\left\{\operatorname{stop\_ ftp}(2)\right\},\{\right.$ block_host $\left.(0)\}\right\}$. After pruning (Line 12), we obtain $\sigma(r \operatorname{sh}(1,2))=$ $\{\{$ block_host $(0)\}\}$, as $\{$ block_host $(0)\}$ is the strategy with the lowest cost. Finally, we obtain $\sigma(\operatorname{root}(2))=$ $\{\{$ block_host $(0)\}\}$, that is the algorithm, in this case, returns the optimal solutions.

From the example above, it is clear that in our approach administrators only have to assign the cost of performing allowable actions (which are meaningful aggregates of initial conditions), whereas in previous approaches they had to assign cost values to each individual initial condition.

Now, let us consider a different example showing how the value of $k$ may have an impact on the optimality of the solution. Intuitively, the higher the value of $k$, the closer the computed solution is to the optimal one.

Example 2: Consider the attack graph of Figure 6, and assume that $\operatorname{cost}\left(\left\{A_{1}\right\}\right)=10, \operatorname{cost}\left(\left\{A_{2}\right\}\right)=18$, and $\operatorname{cost}\left(\left\{A_{3}\right\}\right)=10$. Also assume that cost is additive. It is clear that the optimal strategy to harden the network with respect to $c_{5}$ is $S=\left\{A_{2}\right\}$, with a cost of 18 . Let us now examine the behavior of the algorithm for $k=1$. On Line 1 we obtain $Q=\left\langle c_{1}, c_{2}, c_{3}, c_{4}, e_{1}, e_{2}, c_{5}\right\rangle$. Thus, $c_{1}$ is the 
first node to be examined. After the first 4 elements of the queue have been examined, we obtain $\sigma\left(c_{1}\right)=\left\{\left\{A_{1}\right\}\right\}$, $\sigma\left(c_{2}\right)=\left\{\left\{A_{2}\right\}\right\}, \sigma\left(c_{3}\right)=\left\{\left\{A_{2}\right\}\right\}$, and $\sigma\left(c_{4}\right)=\left\{\left\{A_{3}\right\}\right\}$. Then $e_{1}$ is considered. The full set of possible strategies for $e_{1}$ is $\sigma\left(e_{1}\right)=\left\{\left\{A_{1}\right\},\left\{A_{2}\right\}\right\}$, but, since $k=1$, only the best one is maintained and propagated to following steps. A similar consideration applies to $e_{2}$. In conclusion we obtain $\sigma\left(e_{1}\right)=\left\{\left\{A_{1}\right\}\right\} \sigma\left(e_{2}\right)=\left\{\left\{A_{3}\right\}\right\}$. Finally, we obtain $\sigma\left(c_{5}\right)=\left\{\left\{A_{1}, A_{3}\right\}\right\}$, and $\min \operatorname{Cost}\left(c_{5}\right)=20$, which is slightly above the optimal cost. Similarly, it can be shown that, for $k=2$, the algorithm returns $\min \operatorname{Cost}\left(c_{5}\right)=18$, i.e., the optimal solution. This confirms that larger values of $k$ make solutions closer to the optimal one.

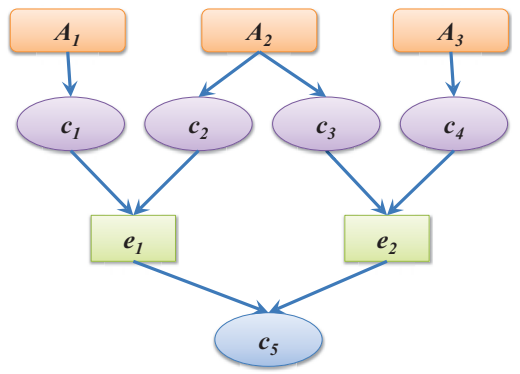

Figure 6. Example of attack graph with $d=2$ and $n=2$

We now show that, in the worst case - when $k=1-$ the approximation ratio is upper-bounded by $n^{d / 2}$. However, experimental results indicate that, in practice, the approximation ratio is much smaller than its theoretical bound. First, let us consider the type of scenario in which solutions may not be optimal. To this aim, consider again the attack graph configuration of Figure 6. When computing solutions for $e_{1}$ and $e_{2}$ respectively, we make local decisions without considering the whole graph, i.e., we independently compute the optimal solution for $e_{1}$ and the optimal solution for $e_{2}$, given hardening strategies for their preconditions. However, at a later stage, we need to merge solutions for both $e_{1}$ and $e_{2}$ in order to obtain solutions for $c_{5}$. At this point, since there exists an allowable action (i.e., $A_{2}$ ) that would have disabled preconditions of both $e_{1}$ and $e_{2}$, with a cost lower than the combined cost of their locally optimal solutions, but the strategy including $A_{2}$ has been discarded for $k=1$, the solution is not optimal. This suggests that both $k$ and the maximum in-degree $n$ of nodes in the graph play a role in determining the optimality of the solution. Additionally, as the algorithm traverses the graph towards target conditions, there may be a multiplicative effect in the approximation error. In fact, the depth $d$ of the tree also plays a role in determining the outcome of the approximation, but this effect can be compensated by increasing the value of $k$. We can prove the following theorem.

Theorem 1: Given an attack graph $G$ with depth $d$ and maximum in-degree $n$, the upper bound of the approxima- tion ratio of algorithm ForwardSearch for $k=1$ is $n^{\frac{d}{2}}$.

Proof: We prove the result by induction, assuming that the cost function is additive. We use the term level $l$ to denote nodes that are at a distance $l$ from the target condition. We need to prove that for each $l \in[1, d-2]$, the cost of hardening conditions at level $l$ is $n^{\frac{d-l}{2}}$ times the optimal cost. The worst case - depicted in Figure 7 is the one in which (i) a single allowable action $A^{*}$ (with $\operatorname{cost}\left(\left\{A^{*}\right\}\right)=x$ ) disables one precondition for each of the $\frac{m}{2}$ exploits $e_{d-1, i}$ at level $d-1$ (i.e., exploits depending on initial conditions), where $m=n^{d}$ is the number of initial conditions; (ii) for each exploit $e_{d-1, i}$, all the preconditions not disabled by $A^{*}$ are disabled by an action $A_{i}$ such that $\operatorname{cost}\left(\left\{A_{i}\right\}\right)=x-\varepsilon$, where $\varepsilon$ is an arbitrarily small positive real number; and (iii) actions $A_{i}$ are pairwise disjoint.

Base case. When choosing a strategy for $e_{d-1, i}$, the algorithm picks the one with the lowest cost, that is strategy $\left\{A_{i}\right\}$ with cost $x-\varepsilon$. Then, when choosing a strategy for $c_{d-2, i}$, the algorithm combines strategies for its $n$ predecessors, which all cost $x-\varepsilon$. Since such strategies are disjoint and cost is additive, the cost to harden any condition at level $d-2$ of the attack tree is $n \cdot(x-\varepsilon)$.

Inductive step. If hardening strategies for conditions at level $d-j$ of the attack tree cost $n^{j / 2} \cdot(x-\varepsilon)$, then hardening strategies for exploits at level $d-j-1$ of the attack tree also $\operatorname{cost} n^{j / 2} \cdot(x-\varepsilon)$. When choosing a strategy for conditions at level $d-j-2$, the algorithm combines strategies for its $n$ predecessors, which all cost $n^{j / 2} \cdot(x-\varepsilon)$. Since such strategies are disjoint and cost is additive, the cost to harden any condition at level $d-j-2$ of the attack tree is $n \cdot n^{j / 2}$. $(x-\varepsilon)=n^{\frac{j+2}{2}} \cdot(x-\varepsilon)$.

Although this result indicates that the bound may increase exponentially with the depth of the attack tree, the bound is in practice - as confirmed by experimental results much lower than the theoretical bound. In fact, the worst case scenario depicted in Figure 7 is quite unrealistic. Additionally, the bound can be reduced by increasing the value of $k$. For instance, by setting $k=n$, the bound becomes $n^{\frac{d-2}{2}}$, that is the bound for a graph with depth $d-2$ and in-degree $n$.

Example 3: Consider the attack graph configuration of Figure 6 (with $n=2$ and $d=2$ ), and assume that $\operatorname{cost}\left(\left\{A_{2}\right\}\right)=x, \operatorname{cost}\left(\left\{A_{1}\right\}\right)=x-\varepsilon$, and $\operatorname{cost}\left(\left\{A_{3}\right\}\right)=$ $x-\varepsilon$. For $k=1$, if the cost function is additive, we obtain $\min \operatorname{Cost}\left(c_{5}\right)=2 \cdot(x-\varepsilon) \approx 2 \cdot x$, which means that in the worst case the cost is twice the optimal cost.

\section{EXPERIMENTAL RESULTS}

In this section, we report the experiments we conducted to validate our approach. Specifically, our objective is to evaluate the performance of algorithm ForwardSearch in terms of processing time and approximation ratio for different values of the depth $d$ of the attack graph and the maximum in-degree $n$ of nodes in the graph. In order to obtain graphs 


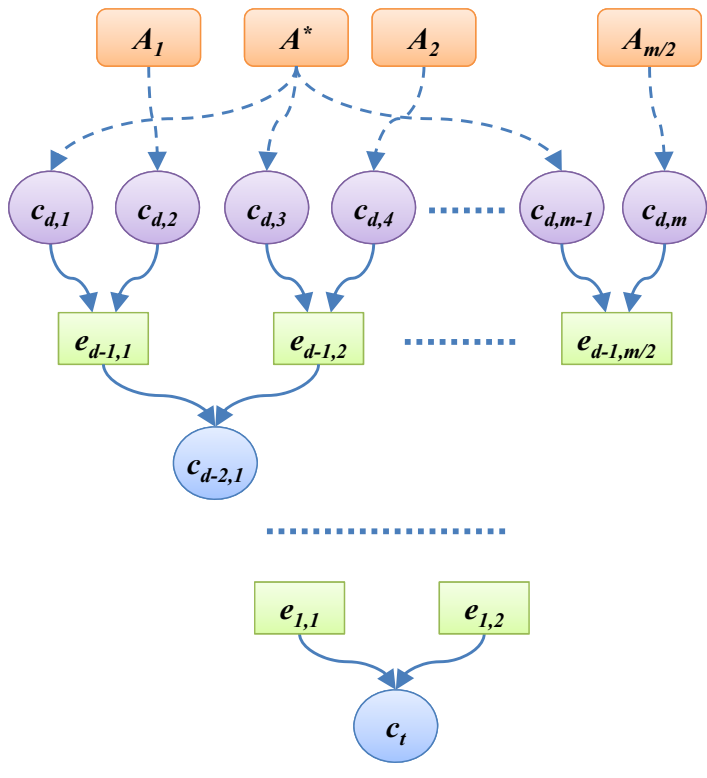

Figure 7. Worst case scenario

with specific values of $d$ and $n$, we started from realistic graphs, like the one of Figure 3, and augmented them with additional synthetic conditions and exploits. Although the large attack graphs we generated through this process are mostly synthetic, we made considerable efforts to make such graphs consistent with real attack graphs. Additionally, for each such graph, we randomly generated different groupings of initial conditions into allowable actions, in order to account for variability in what administrators can control. All the results reported in this section are averaged over multiple graphs with the same values of $d$ and $n$, but different configurations of allowable actions.

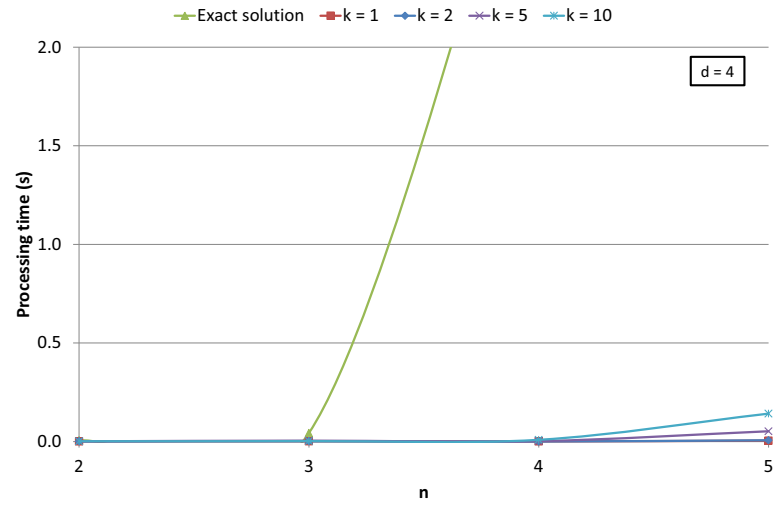

Figure 8. Processing time vs. $n$ for $d=4$ and different values of $k$

First, we show that, as expected, computing the optimal solution is feasible only for very small graphs. Figure 8 shows how processing time increases when $n$ increases and for $d=4$, and compares processing times of the exact algorithm with processing times of algorithm ForwardSearch for different values of $k$. It is clear that the time to compute the exact solution starts to diverge at $n=4$, whereas processing time of algorithm ForwardSearch is still well under 0.5 seconds for $k=10$ and $n=5$. Similarly, Figure 9 shows how processing time increases when $d$ increases and for $n=2$, and compares processing times of the exact algorithm with processing times of algorithm ForwardSearch for different values of $k$. The time to compute the exact solution starts to diverge at $d=5$, whereas processing time of algorithm ForwardSearch is still under 20 milliseconds for $k=10$ and $d=10$.

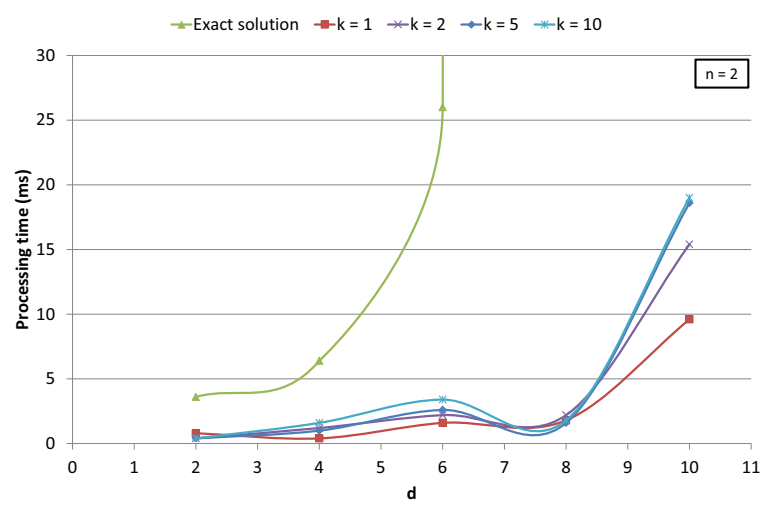

Figure 9. Processing time vs. $d$ for $n=2$ and different values of $k$

Figure 10 shows how processing time increases when the parameter $k$ increases and for a fixed value of $n(n=4)$ and different values of $d$. From this chart, it is clear that large graphs can be processed in a few seconds for values of $k$ up to 5. As we will show shortly, relatively small values of $k$ provide a good balance between approximation ratio and processing time, therefore this result is extremely valuable. Similarly, Figure 11 shows how processing time increases when $k$ increases and for a fixed value of $d(d=8)$ and different values of $n$. This chart confirms that large graphs can be processed in a few seconds for relatively small values of $k$.

We also observed the relationship between processing time and size of the graphs (in terms of number of nodes). Figure 12 shows a scatter plot of average processing times for given pairs of $d$ and $n$ vs. the corresponding graph size. This chart suggests that, in practice, processing time is linear in the size of the graph for small values of $k$.

Finally, we evaluated the approximation ratio achieved by the algorithm. Figure 13 shows how the ratio changes when $k$ increases and for a fixed value of $n(n=2)$ and different values of $d$. It is clear that the approximation ratio improves when $k$ increases, and, in all cases, the ratio is clearly below the theoretical bound. Additionally, relatively 


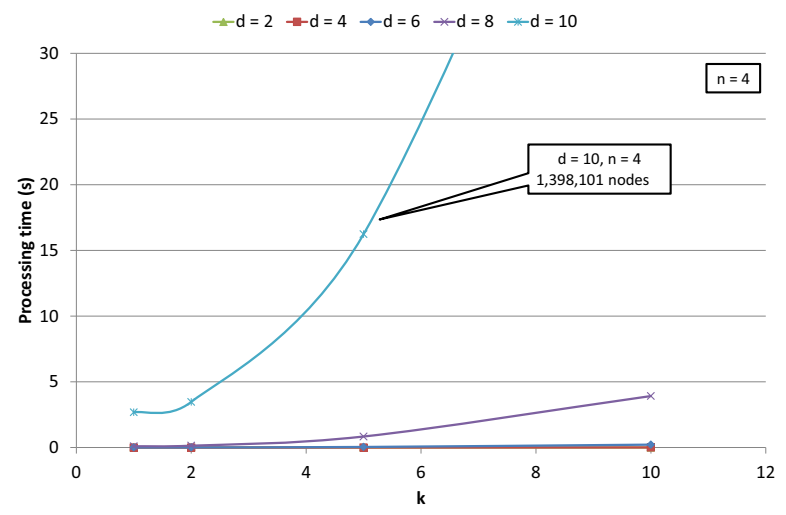

Figure 10. Processing time vs. $k$ for $n=4$ and different values of $d$

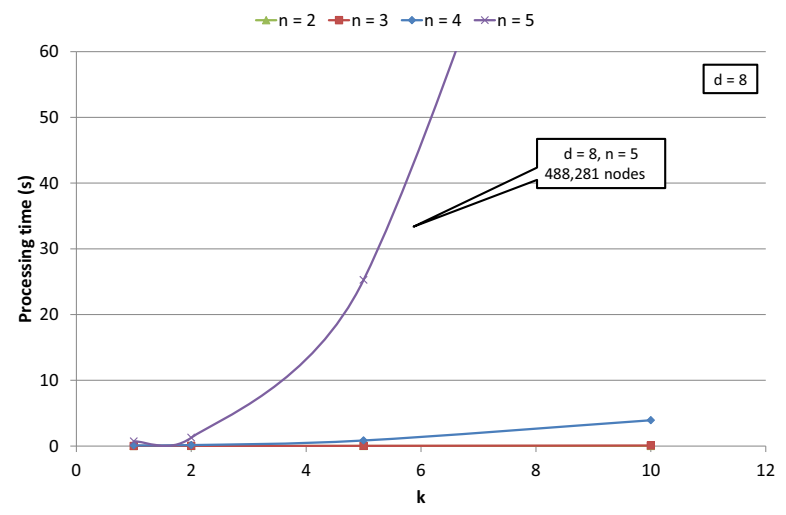

Figure 11. Processing time vs. $k$ for $d=8$ and different values of $n$

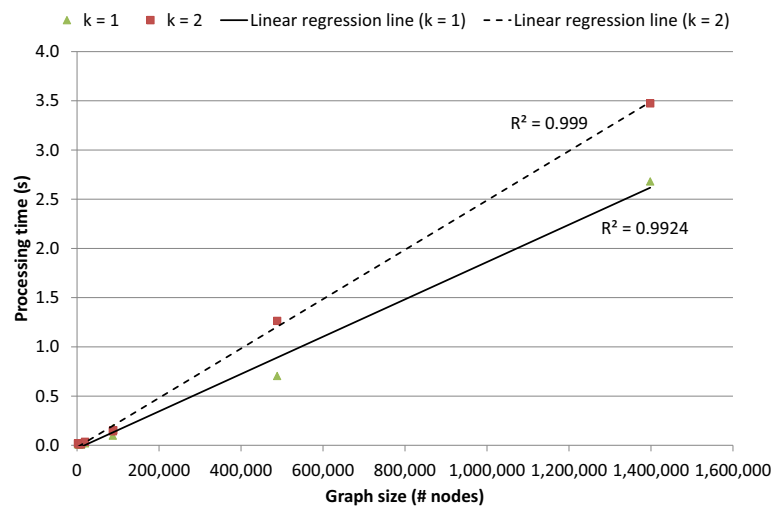

Figure 12. Processing time vs. graph size for different values of $k$

low values of $k$ (between 2 and 6) are sufficient to achieve a reasonably good approximation ratio in a time-efficient manner. Additionally, as observed earlier, processing time is practically linear in the size of the graph for lower values of $k$. Similarly, Figure 14 shows how the approximation ratio
- for a fixed value of $d(d=4)$ and different values of $n$ - improves as $k$ increases. Similar conclusions can be drawn from this chart. In particular, the approximation ratio is always below the theoretical bound.

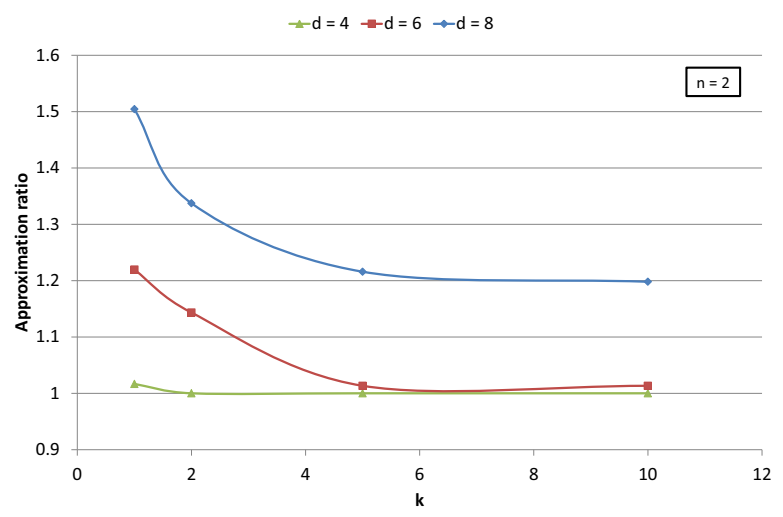

Figure 13. Approximation ratio vs. $k$ for $n=2$ and different values of $d$

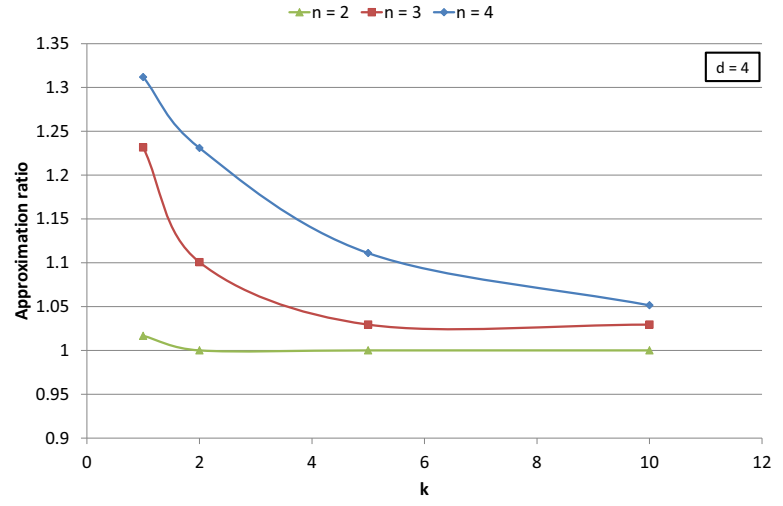

Figure 14. Approximation ratio vs. $k$ for $d=4$ and different values of $n$

\section{CONCLUSIONS}

In this paper, we started by highlighting the limitations of previous work in minimum-cost network hardening using attack graphs. In particular, we showed - both theoretically and experimentally - that finding the exact solution to this problem is feasible only for very small graphs. We proposed an approximation algorithm to find reasonably good solutions in a time-efficient manner. We proved that, under certain reasonable assumptions, the approximation ratio of this algorithm is bounded by $n^{\frac{d}{2}}$, where $n$ is the maximum in-degree of nodes in the graph and $d$ is the depth of the graph. We also showed that, in practice, the approximation ratio is much smaller than its theoretical bound. Finally, we reported experimental results that confirm the validity of our approach, and motivate further research in this direction. 
The experiments described in this paper were conducted on mostly synthetic - yet realistic - attack graphs. Our future plans include evaluating the proposed approach on real data as well as deepening our understanding of cost functions. Although additional work is required, the theoretical and experimental results obtained so far are extremely promising, and the proposed algorithm could be easily adopted to augment the hardening capabilities currently offered by available commercial tools such as Cauldron [18], a vulnerability analysis framework originally developed by members of our research group. Cauldron's current approach to optimal network hardening is based on disabling the smallest possible set of edges in the attack graph, in order to prevent the attacker from reaching certain target conditions. However, this approach has the same limitations of selectively removing exploits. As we discussed earlier in the paper, it is not always possible to remove arbitrary exploits (or attack paths) without removing their causes. In practice, removing sets of initial conditions - which administrators are likely to have control on - will help existing tools with generating hardening recommendations that can be actually enforced.

\section{REFERENCES}

[1] S. Noel and S. Jajodia, "Managing attack graph complexity through visual hierarchical aggregation," in Proceedings of the ACM CCS Workshop on Visualization and Data Mining for Computer Security (VizSEC/DMSEC 2004). Fairfax, VA, USA: ACM, October 2004, pp. 109-118.

[2] L. Wang, S. Noel, and S. Jajodia, "Minimum-cost network hardening using attack graphs," Computer Communications, vol. 29, no. 18, pp. 3812-3824, November 2006.

[3] Tenable Network Security ${ }^{\circledR}$, “The Nessus ${ }^{\circledR}$ vulnerability scanner," http://www.tenable.com/products/nessus.

[4] P. Ammann, D. Wijesekera, and S. Kaushik, "Scalable, graphbased network vulnerability analysis," in Proceedings of the 9th ACM Conference on Computer and Communications Security (CCS 2002), Washington, DC, USA, November 2002, pp. 217-224.

[5] M. Dacier, "Towards quantitative evaluation of computer security," Ph.D. dissertation, Institut National Polytechnique de Toulouse, 1994.

[6] S. Jajodia, S. Noel, and B. O'Berry, Managing Cyber Threats: Issues, Approaches, and Challenges, ser. Massive Computing. Springer, 2005, vol. 5, ch. Topological Analysis of Network Attack Vulnerability, pp. 247-266.

[7] R. Ortalo, Y. Deswarte, and M. Kaâniche, "Experimenting with quantitative evaluation tools for monitoring operational security," IEEE Transactions on Software Engineering, vol. 25, no. 5, pp. 633-650, September/October 1999.

[8] C. Phillips and L. P. Swiler, "A graph-based system for network-vulnerability analysis," in Proceedings of the New Security Paradigms Workshop (NSPW 1998), Charlottesville, VA, USA, September 1998, pp. 71-79.
[9] R. W. Ritchey and P. Ammann, "Using model checking to analyze network vulnerabilities," in Proceedings of the 2000 IEEE Symposium on Research on Security and Privacy ( $\& \& P$ 2000), Berkeley, CA, USA, May 2000, pp. 156-165.

[10] R. Ritchey, B. O'Berry, and S. Noel, "Representing TCP/IP connectivity for topological analysis of network security," in Proceedings of the 18th Annual Computer Security Applications Conference (ACSAC 2002), Las Vegas, NV, USA, December 2002, pp. 25-34.

[11] O. Sheyner, J. Haines, S. Jha, R. Lippmann, and J. M. Wing, "Automated generation and analysis of attack graphs," in Proceedings of the 2002 IEEE Symposium on Security and Privacy ( $\& \& P$ 2002), Berkeley, CA, USA, May 2002, pp. 273-284.

[12] L. P. Swiler, C. Phillips, D. Ellis, and S. Chakerian, "Computer-attack graph generation tool," in Proceedings of the DARPA Information Survivability Conference \& Exposition II (DISCEX 2001), vol. 2, Anaheim, CA, USA, June 2001, pp. 307-321.

[13] D. Zerkle and K. Levitt, "NetKuang - A multi-host configuration vulnerability checker," in Proceedings of the 6th USENIX Security Symposium, San Jose, CA, USA, July 1996.

[14] C. R. Ramakrishnan and R. Sekar, "Model-based analysis of configuration vulnerabilities," Journal of Computer Security, vol. 10, no. 1/2, pp. 189-209, 2002.

[15] S. Jha, O. Sheyner, and J. Wing, "Two formal analyses of attack graphs," in Proceedings of 15th IEEE Computer Security Foundations Workshop (CSFW 2002), Cape Breton, Canada, June 2002

[16] S. Noel, E. Robertson, and S. Jajodia, "Correlating intrusion events and building attack scenarios through attack graph distances," in Proceedings of the 20th Annual Computer Security Applications Conference (ACSAC 2004), Tucson, AZ, USA, December 2004, pp. 350-359.

[17] L. Wang, A. Liu, and S. Jajodia, "Using attack graphs for correlating, hypothesizing, and predicting intrusion alerts," Computer Communications, vol. 29, no. 15, pp. 2917-2933, September 2006.

[18] S. Jajodia, S. Noel, P. Kalapa, M. Albanese, and J. Williams, "Cauldron: Mission-centric cyber situational awareness with defense in depth," in Proceedings of the Military Communications Conference (MILCOM 2011), Baltimore, MD, USA, November 2011 